\title{
Actividad inducida por androsterona y hemisuccinato de androsterona sobre la presión de perfusión y la resistencia vascular
}

\author{
Lauro Figueroa', Francisco Díaz², Abelardo Camacho³, Eliseo Díaz, Rolando Marvin \\ 1 Laboratorio de Fármaco-Química, Facultad de Ciencias Químico-Biológicas, Universidad Autónoma de \\ Campeche, Campeche, México \\ 2 Laboratorio de Química Orgánica, Escuela Nacional de Ciencias Biológicas, Instituto Politécnico \\ Nacional, México, D.F., México \\ ${ }^{3}$ Facultad de Medicina, Universidad Juárez del Estado de Durango, Durango, México
}

Introducción. Existen pocos datos sobre los efectos de la androsterona y sus derivados a nivel cardiovascular. Además, el mecanismo molecular de estos andrógenos y su sitio de acción celular son poco claros.

Objetivo. Evaluar el efecto inducido por la androsterona y el hemisuccinato de androsterona sobre la presión de perfusión y la resistencia vascular.

Materiales y métodos. Los efectos de la androsterona y del derivado de androsterona sobre la presión de perfusión y la resistencia vascular fueron evaluados en un modelo de corazón aislado de rata (Langendorff).

Resultados. Los resultados mostraron que: 1) el hemisuccinato de androsterona $\left[10^{-9} \mathrm{M}\right]$ incrementa la presión de perfusión y la resistencia vascular en comparación con la androsterona $\left.\left[10^{-9} \mathrm{M}\right] ; 2\right)$ los efectos del derivado de androsterona $\left[10^{-9} \mathrm{M}-10^{-5} \mathrm{M}\right]$ sobre la presión de perfusión no fueron inhibidos por indometacina $\left[10^{-6} \mathrm{M}\right]$; 3) la nifedipina $\left[10^{-6} \mathrm{M}\right]$ bloquea los efectos ejercidos por el hemisuccinato de androsterona $\left[10^{-9} \mathrm{M}-10^{-5} \mathrm{M}\right]$ sobre la presión de perfusión, y 4) el efecto del derivado de androsterona $\left[10^{-9} \mathrm{M}-10^{-5} \mathrm{M}\right]$ sobre la presión de perfusión en presencia de flutamida $\left[10^{-6} \mathrm{M}\right]$ fue inhibido.

Conclusiones. Los efectos inducidos por androsterona y hemisuccinato de androsterona sobre la presión de perfusión y la resistencia vascular pueden depender de su estructura química. En el caso de la actividad ejercida por el análogo de androsterona, podría involucrar la interacción del esteroide-receptor androgénico e, indirectamente, la activación del canal de calcio y, consecuentemente, inducir variaciones en la presión de perfusión.

Palabras clave: androsterona, flutamida, perfusión, resistencia vascular, ratas Wistar.

Activity induced by androsterone and hemisuccinate of androsterone on perfusion pressure and vascular resistance

Introduction. Few data exist with respect to the effects of androsterone and their derivatives at cardiovascular level. In addition, the molecular mechanisms and cellular site of action of these androgens are still unclear.

Objective. An evaluation was conducted on the effects induced by androsterone and hemisuccinate of androsterone on perfusion pressure and vascular resistance.

Materials and methods. The effects of both androsterone and hemisuccinate of androsterone on the perfusion pressure and vascular resistance in isolated rat hearts (Langendorff model) were evaluated.

Results. The results showed that: (1) the hemisuccinate of androsterone $\left[10^{-9} \mathrm{M}\right]$ increases the perfusion pressure and vascular resistance in comparison with the androsterone $\left[10^{-9} \mathrm{M}\right]$; (2) the effect of androsterone-derivative $\left[10^{-9} \mathrm{M}-10^{-5} \mathrm{M}\right]$ on perfusion pressure not was inhibited by indometacin $\left[10^{-6} \mathrm{M}\right]$; (3) nifedipine $\left[10^{-6} \mathrm{M}\right]$ blocks the effects exerted by hemisuccinate of androsterone $\left[10^{-9} \mathrm{M}-10^{-5} \mathrm{M}\right]$ on perfusion pressure; and (4) the effect of androsterone-derivative $\left[10^{-9} \mathrm{M}-10^{-5} \mathrm{M}\right]$ on perfusion pressure in presence of flutamide $\left[10^{-6} \mathrm{M}\right]$ was inhibited. 
Conclusions. The effects induced by androsterone and hemisuccinate of androsterone on the perfusion pressure and resistance vascular probably involve the interaction of steroid-receptor androgenic and, indirectly, activation of the calcium channel to induce variations in the perfusion pressure.

Key words: androsterone, flutamide, perfusion, vascular resistance; rats, Wistar.

Las enfermedades cardiovasculares son la principal causa de muerte a nivel mundial (1). Existen varios factores de riesgo como son: tabaquismo, alcoholismo, diabetes, hipertensión y altas concentraciones de lípidos, entre otros, que incrementan la progresión de estas alteraciones cardiovasculares (2).

Además, los estudios epidemiológicos (3-7) y clínicos (8) han demostrado que existen diferencias entre los dos sexos en la incidencia de enfermedades cardiovasculares. Esto se ve reflejado en el hecho de que la mujer premenopáusica tiene una baja incidencia de enfermedades coronarias e infartos al miocardio en comparación con hombres de la misma edad (9-11). En este contexto, se ha observado que los estrógenos endógenos promueven un efecto cardioprotector en mujeres premenopáusicas; sin embargo, su producción es muy baja en la etapa de la menopausia $(12,13)$.

En contraste con los efectos de los estrógenos, se ha postulado que los andrógenos en general son asociados como un factor de riesgo para desarrollar enfermedades cardiovasculares en hombres $(14,15)$. Ejemplo de esto son los estudios realizados por Zumoff et al. (16), los cuales mostraron un incremento en los niveles de androsterona $(71 \mathrm{ng} / \mathrm{dl})$ en hombres después de un infarto en comparación con hombres sanos (57 ng/dl). Otros estudios realizados por Carson et al. (17) demostraron que los incrementos en los niveles de androsterona inducen indirectamente decrementos en la producción

\footnotetext{
Correspondencia:

Lauro Figueroa, Laboratorio de Fármaco-Química, Facultad de Ciencias Químico-Biológicas, Universidad Autónoma de Campeche, Campeche, México, Av. Agustín Melgar s/n entre C-20 y Juande La Barrera, Col. Bellavista C.P. 24039, Campeche, Campeche, México.

Telefono: (981) 8119800 , extensión 73006; fax: (981) 811 9800, extnsión 73002

lauro_1999@yahoo.com
}

Recibido: 30/01/09; aceptado:22/06/09 de colesterol, lipoproteínas de baja densidad y de plaquetas, en hombres antecedentes de infarto al miocardio.

Por otro lado, existen reportes en los que se señala que algunos análogos de androsterona también ejercen un efecto sobre el sistema cardiovascular; ejemplo de esto son los estudios realizados por varios investigadores $(18,19)$, los cuales señalan que incrementos en la concentración de dehidroepiandrosterona pueden favorecer el desarrollo de infartos al miocardio en hombres.

Sin embargo, es importante mencionar que existen datos donde se señalan efectos contrarios a los anteriormente mencionados $(20,21)$; ejemplo de esto son los estudios realizados por Gray et al. (22), en el que se encontró que los bajos niveles de dehidroepiandrosterona pueden predecir enfermedades cardiovasculares en hombres. Además, otros datos señalan que los bajos niveles de dehidroepiandrosterona pueden ser relacionados con hipertensión y obesidad (23), que son dos factores en el desarrollo de enfermedades cardiovasculares. Aunado a esto, otros estudios realizados por Schunkert et al. (24) mostraron una relación positiva entre la presión sanguínea y los niveles de sulfato de dehidroepiandrosterona. Sin embargo, en los estudios realizados por Kawano et al. (25) en hombres con hipercolesteremia, se observó una reducción en la presión sanguínea por incrementos en los niveles del derivado de androsterona.

Por lo anteriormente descrito, los estudios enfocadosaanalizarlosefectos delaandrosterona y sus análogos sobre el aparato cardiovascular que es blanco de su acción, no solamente son escasos sino que han arrojado hasta la fecha resultados que son frecuentemente difíciles de interpretar y a menudo contradictorios. Parte de la dificultad en la interpretación puede ser el abordaje que cada investigador realiza o porque 
el efecto ejercido por la androsterona y sus derivados lo inducen mediante la interferencia con diferentes mecanismos de acción.

Por lo tanto, el presente trabajo tuvo el propósito de evaluar el efecto que ejercen la androsterona y el hemisuccinato de androsterona sobre la capacidad de los vasos coronarios y sobre la resistencia vascular, traducidos por cambios en la presión de perfusión en corazón aislado de rata (modelo de Langendorff) (26). Además, se usaron como herramientas farmacológicas varias sustancias, como la flutamida (antagonista de receptores de andrógenos) (27), la nifedipina (antagonista de canales de calcio tipo-L) (28) y la indometacina (inhibidor de la síntesis de prostanglandinas) (29), para tratar de caracterizar el posible mecanismo involucrado en el efecto ejercido por este andrógeno y su derivado, tal como lo hacen otros tipos de conjugados de andrógenos, que involucran indirectamente la síntesis y liberación de algunas substancias vasoactivas que ejercen indirectamente efectos sobre el tono vascular $(30,31)$.

\section{Materiales y métodos}

\section{Material químico}

El hemisuccinato de androsterona (figura 1) fue sintetizado usando técnicas descritas previamente para hemisuccinatos esteroides (32). La androsterona y los otros reactivos fueron obtenidos de Sigma-Aldrich Chemical Co.

\section{Material biológico}

Todos los protocolos fueron aprobados por el Comité de Ética de la Universidad Autónoma de
Campeche. Su aprobación se basó en la guía para cuidados y uso de animales de laboratorio, publicada por la National Academy of Sciences de los Estados Unidos en 1996 (33). La cepa que se utilizó fue ratas Wistar (con un peso de 220 a $250 \mathrm{~g}$ ), antes de su experimentación se mantuvieron a una temperatura de $22{ }^{\circ} \mathrm{C}$ y fueron alimentadas con una dieta balanceada (purina) y agua para mantenerlas en condiciones deseables.

\section{Diseño experimental}

La población de estudio fue de 90 ratas íntegras (machos, no castradas), de las cuales, se formaron 10 grupos para evaluar el efecto inducido por androsterona y el hemisuccinato de androsterona en un modelo de corazón aislado y perfundido a flujo constante $(10 \mathrm{ml}$ por minuto). Es importante mencionar que cada corazón fue su propio control (para cada serie de experimentos la $\mathrm{n}=9$ ).

\section{Método de Langerdoff}

Los animales fueron anestesiados con pentobarbital por vía intraperitoneal $(50 \mathrm{mg} / \mathrm{kg}$ de peso). Después de la inducción de anestesia, el corazón se extrajo del animal, por medio de una laparatomía toraco-abdominal, y se depositó en un vaso de precipitados que contenía solución de Krebs-Henseleit, la cual estaba compuesta de: $\mathrm{NaCl}(127 \mathrm{mM}), \mathrm{KCl}(6 \mathrm{mM}), \mathrm{CaCl}_{2}(1,8 \mathrm{mM})$, $\mathrm{NaH}_{2} \mathrm{PO}_{4}(1,2 \mathrm{mM}), \mathrm{MgSO}_{4}(1,2 \mathrm{mM}), \mathrm{NaHCO}_{3}$ (25 mM), dextrosa (5 mM), ácido pirúvico (2 mM).

Esta solución se ajustó a un pH de 7,4 (con $\mathrm{NaHCO}_{3}$ ) y se dejó que formara burbujas con

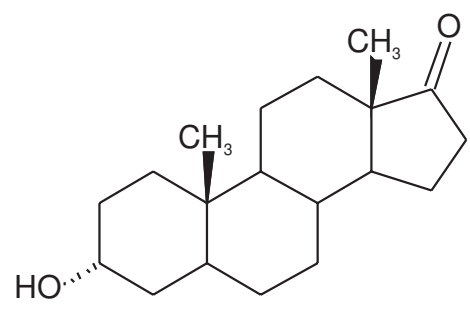

1

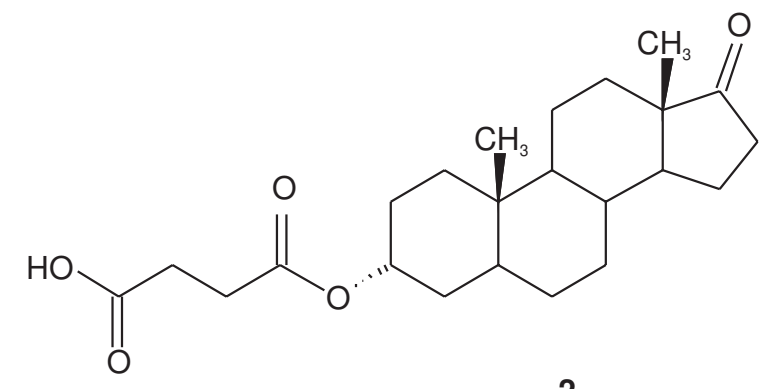

2

Figura 1. Estructura química de la androsterona (1) y el hemisuccinato de androsterona (2). 
una mezcla de gases $\left(5 \% \mathrm{CO}_{2}\right.$ y $\left.95 \% \mathrm{O}_{2}\right)$, a una temperatura de 35 a $36^{\circ} \mathrm{C}$, regulada por un termostato. En este método, el corazón fue perfundido con la solución de Krebs-Henseleit retrógradamente a través de una cánula insertada en la aorta. Aquí es importante mencionar que el tiempo de recuperación del corazón fluctúa entre 10 y 15 minutos. Después de este lapso, se realizó la evaluación farmacológica en tiempos no mayores de una hora.

\section{Presión de perfusión}

Los cambios en la presión de perfusión inducidos por la aplicación de la pregnenolona y el hemisuccinato de pregnenolona, en ausencia y en presencia de las sustancias involucradas en este estudio, fueron cuantificados mediante una derivación de la cánula (la cual estaba insertada en cada corazón) conectada a un transductor de presión (Statham $\AA$ ) que, a su vez, estaba acoplado a un sistema de captura de datos computarizado (Biopac®). Aquí cabe mencionar que la presión de perfusión intraórtica descrita por otros investigadores (34) es de $30 / 40 \mathrm{~mm}$ $\mathrm{Hg}$ en ratas.

\section{Evaluación de los efectos farmacológicos}

Evaluación del efecto inducido por la androsterona $y$ el hemisuccinato de androsterona sobre la presión de perfusión. Se registró la presión de perfusión en tiempos de 3 a 15 minutos en ausencia (control) y en presencia de androsterona y hemisuccinato de androsterona, a dosis de $10^{-9} \mathrm{M}$ (dosis minima). Los esteroides se disolvieron en metanol $1 \mathrm{mg} /$ $\mathrm{ml}$ y las diferentes concentraciones de trabajo se obtuvieron diluyendo esta solución concentrada con solución de $\mathrm{K}-\mathrm{H}(0,01 \%$, v/v).

Determinación del efecto ejercido por androsterona y el hemisuccinato de androsterona sobre la resistencia vascular. Se obtuvo el registro del efecto sobre la resistencia vascular en condiciones de control, en presencia de androsterona y del hemisuccinato de androsterona, a una concentración de $10^{-9} \mathrm{M}$. La androsterona y el derivado de androsterona fueron perfundidos en lapsos no mayores de 15 minutos. La resistencia vascular se calculó dividiendo la presión de perfusión inducida por el flujo coronario entre el valor de flujo que la indujo ( $\mathrm{mm} \mathrm{Hg} / \mathrm{ml}$ por minuto) (30).

Evaluación del efecto del hemisuccinato de androsteronasobrelasíntesis deprostaglandinas. Se administraron diferentes dosis $\left(10^{-9}\right.$ a $\left.10^{-5} \mathrm{M}\right)$ del derivado de androsterona y se registró la presión de perfusión. La curva dosis-respuesta registrada (control) fue repetida bajo la perfusión continua de indometacina $\left(10^{-6} \mathrm{M}\right)$ en el mismo experimento. Aquí cabe mencionar que la duración de la preincubación con indometacina fue de 10 minutos.

Efecto del hemisuccinato de androsterona sobre la presión de perfusión mediado a través de canales de calcio (tipo-L). Se administraron diferentes dosis del análogo de androsterona $\left(10^{-9}\right.$ a $\left.10^{-5} \mathrm{M}\right)$ y se registró la presión de perfusión. La curva dosis-respuesta (control) fue repetida bajo perfusión continua de nifedipina $\left(10^{-6} \mathrm{M}\right)$ en el mismo experimento. Aquí cabe señalar que la duración de la preincubación con nifedipina fue de 10 minutos.

Determinación del efecto inducido por el hemisuccinato de androsterona sobre la presión de perfusión por medio del receptor androgénico. Se administraron diferentes dosis $\left(10^{-9}\right.$ a $\left.10^{-5} \mathrm{M}\right)$ del análogo de androsterona y se registró la presión de perfusión. La curva dosis-respuesta registrada (control) fue repetida bajo perfusión continua de flutamida $\left(10^{-6} \mathrm{M}\right)$ en el mismo experimento. Es importante mencionar que la duración de la preincubación con flutamida fue de 10 minutos.

Es importante mencionar que la dosis $\left(10^{-6}\right.$ M) administrada de los compuestos como indometacina, nifedipina y flutamida, usados en este estudio, se determinó con base en estudios realizados anteriormente para otros esteroides $(26,29)$ y en las curvas dosis-respuesta (no mostradas) de cada uno de ellos en presencia del hemisuccinato de androsterona.

\section{Análisis estadístico}

Los valores obtenidos son expresados como media \pm E.E.M. y los datos obtenidos fueron sometidos a prueba $\mathrm{T}$ de Student y análisis de 
varianza (ANOVA) con factor de corrección de Bonferroni (35). Las diferencias se consideraron significativas cuando $p$ tuvo un valor igual 0 menor a 0,05.

\section{Resultados}

En este estudio se evaluó la actividad biológica de la androsterona y su derivado sobre la presión de perfusión. Los resultados obtenidos señalan que con el incremento en el tiempo (3 a 15 minutos), en condiciones de control $(43,84$ a $45,47 \mathrm{~mm}$ $\mathrm{Hg}$ ) y en presencia de androsterona $\left(10^{-9} \mathrm{M}\right)$, la presión de perfusión no varía significativamente (44,50 a 45,80 mm Hg) (figura 2). Sin embargo, en presencia del derivado de androsterona $\left(10^{-9}\right.$ $M)$ se incrementa significativamente $(48,57$ a $53,00 \mathrm{~mm} \mathrm{Hg})(p=0,005)$. Además, los datos registrados en la evaluación del efecto del flujo coronario sobre la resistencia vascular (figura 3) en ausencia, así como en presencia de androsterona y el hemisuccinato de androsterona a una concentración de $\left(10^{-9} \mathrm{M}\right)$, muestran

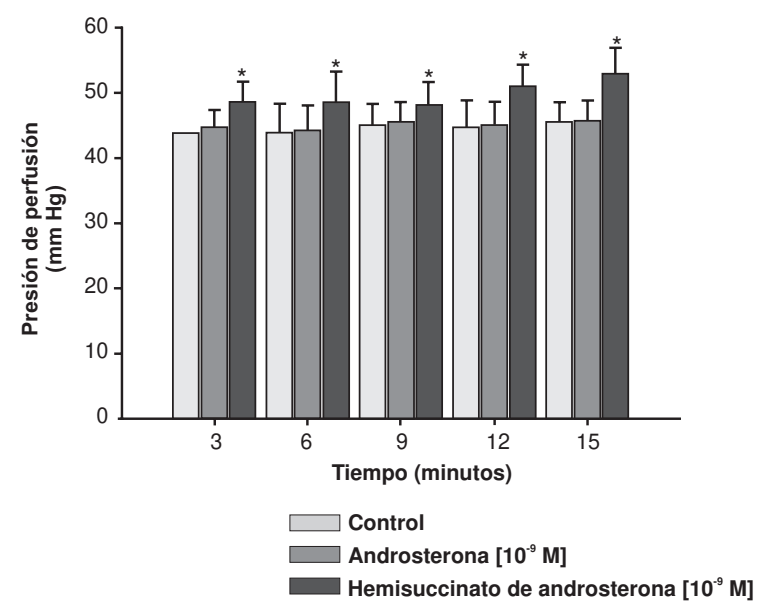

Figura 2. En la gráfica se muestra el efecto ejercido por la androsterona y el hemisuccinato de androsterona sobre la presión de perfusión en un modelo de rata aislada y perfundido a flujo constante (Langendorff). Los resultados obtenidos señalan que el incremento en el tiempo (3 a 15 minutos) en condiciones de control y en presencia de androsterona (10-9 M) la presión de perfusión no varía significativamente; sin embargo, en presencia del derivado de androsterona (10-9 M), se incrementa significativamente $(p=0,005)$. Es importante señalar que los efectos se expresan como área bajo la curva y cada barra representa la media \pm E.E.M. de nueve experimentos.

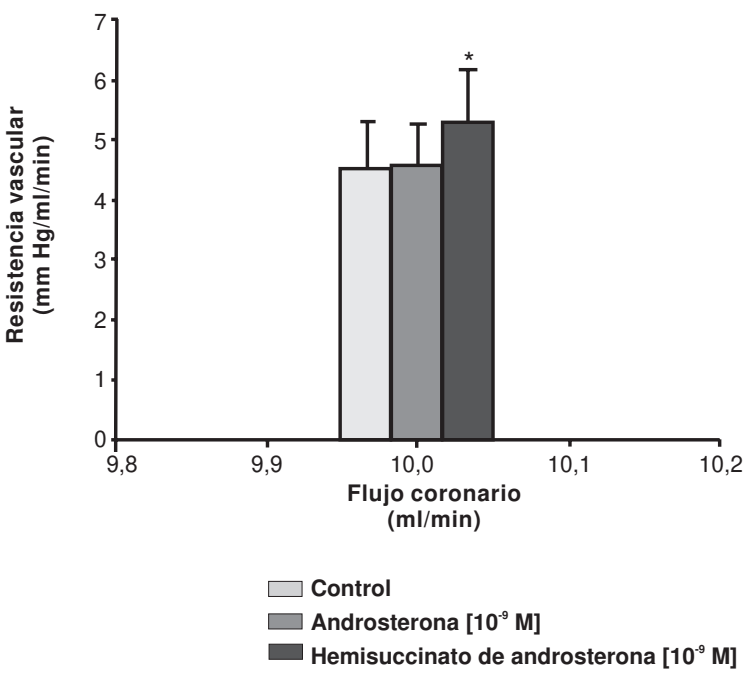

Figura 3. La gráfica señala el registro del efecto ejercido por androsterona y el hemisuccinato de androsterona sobre la resistencia vascular. Los resultados indican que el derivado de androsterona incrementa (10-9 M) significativamente $(p=0,008)$ la resistencia vascular en comparación con la androsterona (10-9 M) y las condiciones control. Aquí es importante señalar que los efectos se expresan como área bajo la curva y cada barra representa la media \pm E.E.M. de nueve experimentos.

que el derivado de androsterona incrementa significativamente $(p=0,008)$ la resistencia vascular en comparación con la androsterona y las condiciones de control.

Otros datos obtenidos en experimentos alternativos, donde se evaluó el efecto del hemisuccinato de androsterona sobre la presión de perfusión en ausencia y en presencia de indometacina $\left(10^{-6} \mathrm{M}\right)$, mostraron que el derivado de androsterona incrementa la presión de perfusión (figura 4) en forma dependiente de la dosis $\left(10^{-9} \mathrm{M}\right.$ a $\left.10^{-5} \mathrm{M}\right)$. Sin embargo, en presencia del inhibidor de la ciclooxigenasa, este efecto no es inhibido.

Por otro lado, los resultados cuando se evaluó el hemisuccinato de androsterona sobre la presión de perfusión en ausencia y en presencia de nifedipina $\left(10^{-6} \mathrm{M}\right)$, señalan que el efecto inducido por el hemisuccinato de androsterona $\left(10^{-9} \mathrm{M}\right.$ a $\left.10^{-5} \mathrm{M}\right)$ sobre la presión de perfusión es bloqueado significativamente $(p=0,005)$ por el antagonista del calcio (figura 5). 
- Control

o Hemisuccinato de androsterona + indometacina $\left[10^{-6} \mathrm{M}\right]$

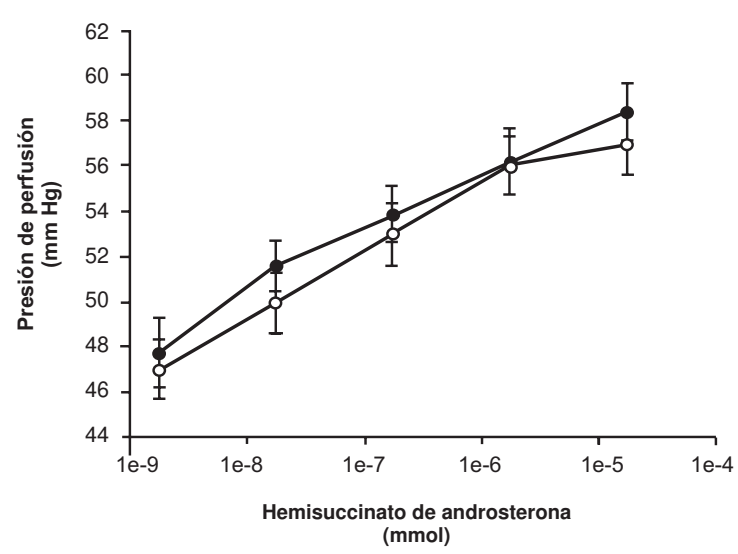

Figura 4. Evaluación del efecto del hemisuccinato de androsterona sobre la síntesis de prostaglandinas. Los registros obtenidos muestran que el derivado de androsterona $(\bullet)$ incrementa la presión de perfusión en una manera que depende de la dosis. Además, en la gráfica se observa que, en presencia del inhibidor de la ciclooxigenasa (o), este efecto no es inhibido. Es importante señalar que los efectos se expresan como área bajo la curva y cada punto representa la media \pm E.E.M. de nueve experimentos.

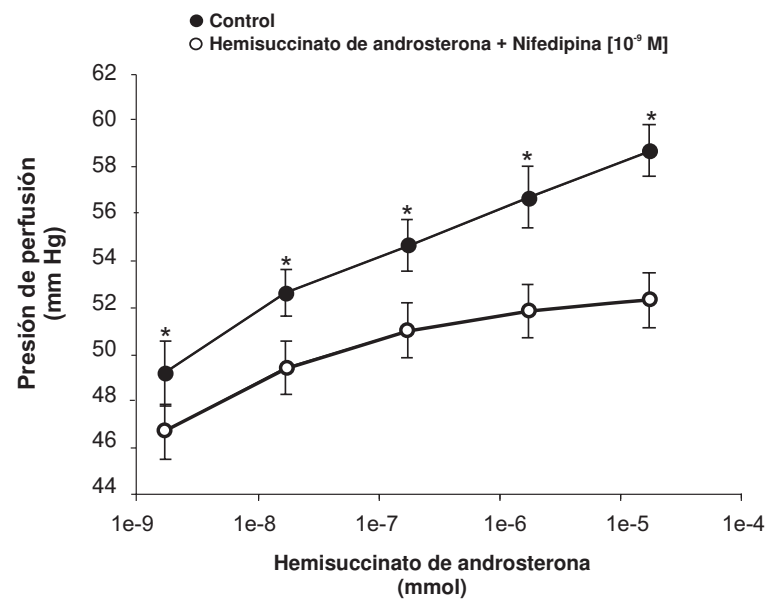

Figura 5. Efecto del hemisuccinato de androsterona sobre la presión de perfusión mediado a través de canales de calcio. Los resultados señalan que el efecto inducido por el análogo de androsterona $(\bullet)$ incrementa la presión de perfusión; sin embargo, este efecto es bloqueado significativamente $(p=0,008)$ por la nifedipina (o). Aquí es importante señalar que los efectos se expresan como área bajo la curva y cada barra representa la media \pm E.E.M. de nueve experimentos.

Por último, los datos obtenidos en la evaluación del efecto del hemisuccinato de androsterona en presencia y en ausencia de flutamida $\left(10^{-6}\right.$

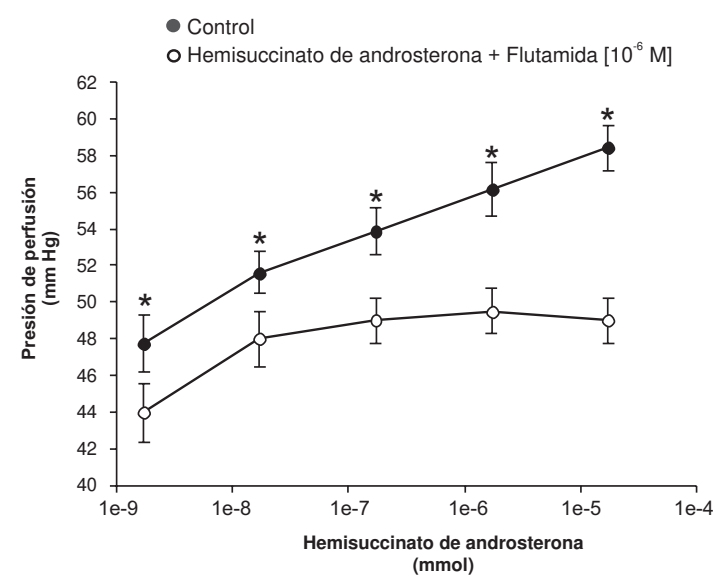

Figura6. Determinación del efecto inducido porhemisuccinato de androsterona sobre la presión de perfusión a través del receptor androgénico. Los datos obtenidos señalan que el aumento en la dosis (10-9 $\mathrm{M}$ a 10-5 M) del análogo de androsterona $(\bullet)$ trae como consecuencia incrementos en la presión de perfusión; sin embargo, en presencia del antagonista de flutamida (o), este efecto disminuye significativamente $(p=0,005)$. Es importante señalar que los efectos se expresan como área bajo la curva y cada barra representa la media \pm E.E.M. de nueve experimentos.

$M)$, señalan que el aumento en la dosis $\left(10^{-9}\right.$ $\mathrm{M}$ a $10^{-5} \mathrm{M}$ ) del análogo de androsterona trae como consecuencia incrementos en la presión de perfusión (figura 6). Sin embargo, este efecto disminuye en presencia del antagonista de andrógenos $(p=0,005)$.

\section{Discusión}

En este trabajo se evaluó el efecto inducido por la androsterona y el hemisuccinato de androsterona sobre la capacidad de los vasos coronarios y sobre la resistencia vascular, traducido por cambios en la presión de perfusión en corazón aislado de rata en un modelo de Langendorff (26). Vale la pena mencionar que nos pareció importante sólo mostrar la actividad ejercida por el hemisuccinato de androsterona a una concentración de $10^{-9} \mathrm{M}$ (dosis minima efectiva). Los resultados obtenidos señalan que la presión de perfusión en condiciones control y en presencia de androsterona $\left(10^{-9}\right.$ M) no cambia con variaciones en el tiempo (1 a 15 minutos). Sin embargo, en presencia del análogo de androsterona $\left(10^{-9} \mathrm{M}\right)$, se incrementa significativamente. 
Estos datos indican que el hemisuccinato de androsterona ejerce mayor efecto sobre la presión de perfusión en comparación con la androsterona, lo que podría traer como consecuencia modificaciones en el tono vascular. Pensando en esto, se analizó el efecto inducido por este andrógeno y su derivado sobre la resistencia vascular, traducido en cambios en la presión de perfusión. Los resultados obtenidos indican que la resistencia vascular se incrementa significativamente en presencia del hemisuccinato de androsterona, en comparación con las condiciones de control y la androsterona.

Estos datos son similares a los encontrados para otro tipo de conjugados esteroidales y que involucran variaciones en la presión de perfusión y la resistencia vascular $(30,31)$.

Sin embargo, hay que recalcar que los grupos funcionales contenidos en la estructura química de estos esteroides son diferentes a los involucrados en el hemisuccinato de androsterona. Por lo tanto, estos datos sugieren que el solo hecho de cambiar la funcionalidad estructural de la androsterona en el anillo $A$ para producir el hemisuccinato de androsterona, trae como consecuencia que este andrógeno induzca un mayor efecto sobre el tono vascular que la androsterona, lo que podría condicionar la síntesis y liberación de algunas substancias vasodilatadoras o vasoconstrictoras involucradas en la regulación del tono vascular (36), como lo hacen otro tipo de esteroides androgénicos que tienen influencia sobre sustancias vasoconstrictoras, específicamente sobre la síntesis de prostaglandinas (30).

Para probar esta hipótesis, en este trabajo se evalúo el efecto inducido por el hemisuccinato de androsterona en ausencia y en presencia de indometacina (antagonista de la ciclooxigenasa) (29) sobre la presión de perfusión, para saber si este derivado androgénico pudiera tener influencia sobre la liberación o síntesis de estas sustancias vasoconstrictoras, como es el caso del tromboxano $A_{2}$ generado en la cascada de las prostaglandinas (37).

Los resultados indican que, en presencia de indometacina, el efecto ejercido por el hemisuccinato de androsterona sobre la presión de perfusión no es bloqueado. Estos datos sugieren que ésta no es la vía por el cual el análogo de androsterona ejerce su actividad sobre la presión de perfusión, por lo que asumimos que el efecto vasopresor inducido por la aplicación del hemisuccinato de androsterona podría involucrar otro mecanismo. En este sentido, se analizaron algunos datos que existen en los que se señala que algunos andrógenos inducen su efecto por medio de la activación de canales de calcio tipo-L $(38,39)$ y, consecuentemente, ejercen su efecto vasopresor sobre la presión sanguínea (23).

Por lo tanto, para evaluar esta hipótesis, en este trabajo se determinó el efecto inducido por el derivado androgénico sobre la presión de perfusión en ausenciay en presencia de nifedipina $\left(10^{-6} \mathrm{M}\right)$. Los resultados mostraron que el efecto ejercido por el hemisuccinato de androsterona en dosis de $10^{-9} \mathrm{M}$ a $10^{-5} \mathrm{M}$ sobre la presión de perfusión, es inhibido significativamente por la presencia de nifedipina. Estos datos indican que el análogo de androsterona podría indirectamente tener influencia sobre los canales de calcio tipo-L, lo que traería como consecuencia incrementos en el calcio intracelular y subsecuentemente en el estado vasopresor inducido por este derivado hormonal.

Es importante mencionar que este fenómeno podría estar condicionado indirectamente por la activación del receptor de andrógenos, como sucede en otro tipo de hormonas andrógenicas (40). Ejemplo de esto son los estudios realizados por Fikret et al. (41), quienes demostraron que algunos andrógenos ejercen sus efectos por medio de la activación del canal de calcio tipo-L, por la vía de interacción con el receptor de andrógenos.

Sin embargo, es conveniente mencionar que dicha activación-interacción del receptor con el esteroide androgénico, depende de los grupos funcionales contenidos en su estructura química, así como del acoplamiento del sistema anular (ciclopentanoperhidrofenantreno) del esteroide con su receptor (42), lo que trae como consecuencia un efecto específico. No obstante, 
es importante mencionar que el mecanismo molecular por el cual los andrógenos, en general, ejercen su efecto, podría ser diferente al mecanismo involucrado en el efecto inducido por el hemisuccinato de androsterona.

Para evaluar esta premisa, se estudió la actividad inducida por el hemisuccinato de androsterona sobre la presión de perfusión, usando como herramienta farmacológica a la flutamida, que es un potente antagonista de receptores de andrógenos (27). Los resultados obtenidos por la aplicación del análogo de androsterona, mostraron que la presión de perfusión aumenta, aunque, en presencia de flutamida, este efecto se ve inhibido significativamente. Todos estos datos indican que la actividad vasopresora inducida por el hemisuccinato de androsterona sobre la presión de perfusión podría involucrar la interacción del esteroide-receptor androgénico e indirectamente activación del canal de calcio tipo-L, lo que puede trae como consecuencia incremento en la concentración de calcio intracelular e, indirectamente, aumento de la presión de perfusión.

En conclusión, los datos obtenidos hacen pensar que los efectos inducidos por androsterona y hemisuccinato de androsterona sobre la presión de perfusión y la resistencia vascular, podrían depender de su estructura química. Además, la actividad ejercida por el análogo de androsterona podría involucrar la interacción del esteroidereceptor androgénico e, indirectamente, activación del canal de calcio (tipo-L) y consecuentemente inducir variaciones en la presión de perfusión. Sin embargo, debido a los tiempos de respuesta del efecto inducido por el hemisuccinato de androsterona, posiblemente podría involucrar un efecto no genómico. Esta hipótesis contrasta con los efectos ejercidos por otros esteroides $(26,30,31)$, que incrementan el calcio intracelular e inducen variaciones en la presión de perfusión por la activación del receptor androgénico a nivel no genómico.

\section{Agradecimientos}

Lauro Figueroa agradece a Angélica León por su valiosa asistencia.

\section{Conflicto de intereses}

No se tiene ningún conflicto de intereses con autoridades públicas o privadas para su publicación.

\section{Financiación}

Este proyecto fue financiado por CONACyT con número de registro 52542.

\section{Referencias}

1. Yusuf S, Reddy S, Ôunpuu S, Anand S. Clinical cardiology: New frontiers. Global burden of cardiovascular diseases. Part I: General considerations, the epidemiologic transition, risk factors and impact of urbanization. Circulation. 2001;104:2746.

2. van den Hoogen $P$, Feskens $E$, Nagelkerke N, Menotti A, Nissinen A, Kromhout D. The relation between blood pressure and mortality due to coronary heart disease among men in different parts of the world. $\mathrm{N}$ Engl J Med. 2000;342:1675-6.

3. Roeters van-Lennep J, Westerveld H, Willem D, Vander E. Risk factors for coronary heart disease: implications of gender. Cardiovasc Res. 2002;53:538-49.

4. Appel H, Weicker $\mathbf{H}$. Ultrastructural and morphometric investigations on the effects of training and administration of anabolic steroids on the myocardium of guinea pigs. Int J Sports Med. 1983;4:268-74.

5. Gordon T, Kannel W. Premature mortality from coronary heart disease: the Framingham Study. J Am Med Assoc. $1971 ; 215: 1617-25$

6. Kentz M, Lugani K. Morbidity and mortality chartbook on cardiovascular, lung and blood diseases. Bethesda, MD: National Heart, Lung, and Blood Institute; 1992. p. $1-55$.

7. Boysen G, Nyboe J. Incidence and risk factors for stroke in Copenhagen, Denmark. Stroke. 1988;19:1345-53.

8. Hippisley-Cox J, Pringle M, Crown N, Meal A, Wynn A. Sex inequalities in ischaemic heart disease in general practice: cross sectional survey. British Med J. 2001;322:832.

9. Rossouw J, Prentice R, Manson J, Wu L, Barad D, Barnabei V. Postmenopausal hormone therapy and risk of cardiovascular disease by age and years since menopause. J Am Med Assoc. 2007;297:1465-77.

10. Kannel G, Hjortland M, McNamara P. Menopause and risk of cardiovascular disease: the Framingham Study. Ann Intern Med. 1976;85:447-52.

11. Stampfer M, Colditz G, Willett W. Postmenopausal estrogen therapy and cardiovascular disease: ten-year follow-up from the Nurses' Health Study. N Engl J Med. 1991;325:756-62. 
12. Grodstein F, Manson J, Stampfer M. Postmenopausal hormone use and secondary prevention of coronary events in the Nurses' Health Study: A prospective, observational study. Ann Intern Med. 2001;135:1-8.

13. Cauley J, Gutai J, Kuller L. The epidemiology of serum sex hormones in postmenopausal women. Am J Epidemiol. 1989;129:1120-31.

14. Liu P, Death A. Handelsman D. Androgens and cardiovascular disease. Endocr Rev. 2003;24:313-40.

15. Levy D, Kannell W. Cardiovascular risks: New insights for Framingham. J Am Heart. 1988;116:266-72.

16. Zumoff B, Troxler R, O'Connor J, Rosenfeld R, Kream J, Levin J. Abnormal hormone levels in men with coronary artery disease. Arteriosclerosis. 1982;2:58-67.

17. Carson P, McDonald L, Pickard S, Pilkington T, Davies B. Effects of clofibrate with androsterone (atromid) and without androsterone (atromid-S) on blood platelets and lipids in ischaemic heart disease. Brit Heart $\mathrm{J}$. 1966;28:400-3.

18. Melentyev A. Psyological features of patients with ischaemic heart disease and coronary behavior type "A". Arteriosclerosis. 2000;151:225-6.

19. Nestler J, Usiskin K, Barlascini C, Welty D, Clore J, Blackard W. Suppression of serum dehydroepiandrosterone sulfate levels by insulin: an evaluation of possible mechanisms. J Clin Endocrinol Metab. 1989;69:1040-6.

20. Ding A, Stallone J. Testosterone-induced relaxation of rat aorta is androgen structure specific and involves $\mathrm{K}^{+}$ channel activation. J Appl Physiol. 2001;91:2742-50.

21. Slowinska-Srzednicka J, Zgliczynski S, Soszynski P, Makowska A, Zgliczynski W, Srzednicki M, et al. Decreased plasma levels of dehydroepiandrosterone sulphate (DHEA-S) in normolipidaemic and hyperlipoproteinaemic young men with coronary artery disease. J Intern Med. 1991;230:551-3.

22. Gray A, Feldman H, Mckinlay J, Longcope C. Age, disease, and changing sex hormone levels in middle-age men: results of the Massachusetts Male Aging Study. J Clin Endocrinol Metab. 1991;73:1016-25.

23. Nestler J, Beer N, Jakubowicz D, Colombo C, Beer R. Effects of insulin reduction with benfluorex on serum dehydroepiandrosterone (DHEA), DHEA sulfate, and blood pressure in hypertensive middle-aged and elderly men. J Clin Endocrinol Metab. 1995;80:700-6.

24. Schunkert H, Hense H, Andus T, Riegger G, Straub R. Relation between dehydroepiandrosterone sulfate and blood pressure levels in a population-based sample. Am J Hypertens. 1999;12:1140-3.

25. Kawano H, Yasue H, Kitagawa A, Hirai N, Yoshida $\mathrm{T}$, Soejima $\mathrm{H}$, et al. Dehydroepiandrosterone supplementation improves endothelial function and insulin sensitivity in men. J Clin Endocrinol Metab. 2003:88:3190-5.

26. Ceballos G, Figueroa L, Rubio I, García A, Martínez A, Yañez R. Acute and nongenomic effects of testosterone on isolated and perfused rat heart. $\mathrm{J}$ Cardiovasc Pharmacol. 1999;33:691-7.

27. Kontula K, Seppanen P, Duyne P, Bardin C, Janne O. Effect of a nonsteroidal antiandrogen, flutamide, on androgen receptor dynamics and ornithine decarboxylase gene expression in mouse kidney. Endocrinology. 1985;116:226-33.

28. Henry PD. Comparative pharmacology of calcium antagonists: nifedipine, verapamil and diltiazem. Am J Cardiol. 1980;46:1047-58

29. Yamamoto T, Nozaki-Taguchi $\mathbf{N}$. Analysis of the effects of cyclooxygenase (COX)-1 and COX-2 in spinal nociceptive transmission using indomethacin, a nonselective COX inhibitor, and NS-398, a COX-2 selective inhibitor.Brain Res. 1996;739:104-10.

30. Figueroa-Valverde L, Luna H, Castillo-Henkel C, Muñoz-García O, Morato-Cartagena T, CeballosReyes G. Synthesis and evaluation of the cardiovascular effects of two, membrane impermeant, macromolecular complexes of dextran-testosterone. Steroids. 2002; 67: 611-9.

31. Figueroa-Valverde L, Díaz-Cedillo F, Diaz-Ku E, Camacho-Luis A. Effect induced by hemisuccinate of pregnenolone on perfusion pressure and vascular resistance in isolated rat heart. African $\mathrm{J}$ Pharm Pharmacol. 2009;3:234-41.

32. Figueroa-Valverde L, Díaz-Cedillo F, Tolosa L, Maldonado M, Ceballos G. Synthesis of pregnenolonepregnenolone dimer via ring A-ting a connection. J Mex Chem Soc. 2006;50:42-5.

33. Institute of Laboratory Animal, Resources Commission on Life Sciences, National Research Council. Guide for the care and use of laboratory animals. Seventh edition. Washington, D.C.: National Academies Press; 1996. p. 1-240.

34. Neely J, Liebermeister H, Battersby E, Morgan H. Effect of pressure development on oxygen consumption by isolated rat heart. Am J Physiol. 1967;212:804-14.

35. Hocht C, Opezzo J, Gorzalczany S, Bramuglia G, Tiara C. Una aproximación cinética y dinámica de metildopa en ratas con coartación aórtica mediante microdiálisis. Rev Argent Cardiol. 1999;67:769-73.

36. Mombouli J, Vanhoutte P. Endothelial dysfunction: a novel therapeutic target endothelial dysfunction: From physiology to therapy. J Mol Cell Cardiol. 1999;31:61-74.

37. Schork M, Morinelli A, Masuda A. Testosterone treatment enhances thrombosane $A_{2}$ mimetic. Induced coronary artery vasoconstriction in guinea pigs. Eur $\mathrm{J}$ Clin Invest. 1994: 24(Suppl.1);50-2. 
38. Pham T, Robinson R, Danilo P, Rosen M. Effects of gonadal steroids on gender-related differences in transmural dispersion of L-type calcium current. Cardiovasc Res. 2002;53:752-62.

39. Guo Z, Peter W, Benten M, Krücken J, Wunderlich F. Nongenomic testosterone calcium signaling, genotropic actions in androgen receptor-free macrophages. J Biol Chem. 2002;277:29600-7.

40. Reckelhoff J, Zhang H, Srivastava K, Granger J. Gender differences in hypertension in spontaneously hypertensive rats. Role of androgens and androgen receptor. Hypertension. 1999;34:920-3.

41. Er F, Michels G, Brandt M, Khan I, Haase H, Eicks M, et al. Impact of testosterone on cardiac L-type calcium channels and $\mathrm{Ca}^{2+}$ sparks: Acute actions antagonize chronic effects. Cell Calcium. 2007;41:467-77.

42. Wolff E, Ho W, Kwok R. The steroid-receptor complex. Some considerations based on $\mathrm{sp}^{2}$-hybrized systems. J Med Chem. 1964;7:77-89. 